\title{
Uso de Internet y su Influencia en el Desempeño Académico de los Estudiantes de la Facultad de Ciencias Agrícolas de la UNJBG
}

\author{
Using of The Internet and its Influence on the Academic \\ Performance of Students of the Faculty of Agricultural Sciences
}

\author{
' Francisco Teodoro Condori Tintaya
}

\begin{abstract}
RESUMEN:
En la actualidad vivimos en una sociedad de la información, una sociedad "audiovisual teleinteractiva" en la que cada vez cobra más importancia la informática. las telecomunicaciones y la comunicación audiovisual, por tanto, requerimos hacer más tareas ante un ordenador multimedia conectado a Internet. En este contexto, fue necesario conocer el nivel de destreza de nuestros estudiantes y profesores de la Facultad de Ciencias Agricolas de la UNJBG en el manejo y uso de la tecnología informática como internet (instrumento didáctico), y equipos de comunicación audiovisual. Para desarrollar esta investigación, se ha tomado como universo a estudiantes de la Facultad de Ciencias Agrícolas $(N=557)$. A través de un muestreo aleatorio simple se ha tomado una muestra $n=221$, estratificado por años de estudio y Escuelas Profesionales. Los resultados indican que, el 65\% de los estudiantes tienen computadoras propias, de los cuales solo el $47 \%$ están conectados a internet; en cuanto al uso de equipos informáticos para su formación profesional, el $67 \%$ solo utiliza procesadores de textos y hoja de cálculos y solo el $5 \%$ utiliza algún paquete estadistico.
\end{abstract}

Palabras Clave: internet, red de redes, calidad de la educación, navegadores. tecnologias de la información y comunicación

\section{ABSTRACT:}

These days, we live in an information society, a "andiovisual teleinteractive" society, where informatics, the telecommunication, and audiovisual communication become more and more important, therefore, we require to do more tasks in front of multimedia computer connected to internet. In this context, it was necessary to know the level of our students and teachers skills in the Faculty of Agricultural Sciences of the UNJBG managing and using the computer technology such as intemet (teaching tool), and audiovisual communications equipment. To develope this investigation, taken were the students of the Faculty of Agricultural Sciences as the whole universo $(N=557)$, through a simple random sample it has been taken a sample $(n=221)$, which is stratified for years of study and Profesional Schools. The results indicate that, $65 \%$ of students have their own computers, but from these only $47 \%$ are connected to internet; about the use of computer equipement 10 their vocational training, $67 \%$ use text processor and calculation sheets and only the $5 \%$ uses any statistical package.

Keywords: Internet, network of networks, quality of education, browsers, information technology and communication 


\section{INTRODUCCIÓN}

Internet en los últimos años, se ha convertido en una poderosa herramienta para ayudar a la difusión del conocimiento y la educación, de hecho es una de las mayores fuentes de información disponibles para los estudiantes en general. Se dice que estamos en la era de la comunicación y el conocimiento, de ahí la importancia de esta red de redes que actualmente se extiende por todas partes del mundo, reduciendo considerablemente el tiempo y esfuerzo empleado en la búsqueda del saber y la información.

Numerosos son los servicios ofrecidos por Internet. entre muchas de ellas tenemos: bibliotecas en lineas, educación a distancia, búsqueda de información, correo electrónico, transferencia de archivos, listas de correo y foros de discusión, videoconferencias, software, boletines electrónicos, entre otros; Por tanto, el uso de Internet en la educación superior está fomentado sobre la base del innegable impacto que ha tenido en el presente y que tendrá en el futuro.

La Universidad Nacional Jorge Basadre Grohmann, y en particular la Facultad de Ciencias Agricolas son actores directamente involucrados con el desarrollo de la ciencia y la tecnologia para contribuir al crecimiento y desarrollo de nuestra región; sin embargo, estos propósitos no serian posibles lograr si es que no se dispone de una herramienta efícaz como es la tecnología de la información y comunicación (TIC).

La presente investigación se ha desarrollado con el propósito de conocer el nivel de destreza de los estudiantes y profesores de la Facultad de Ciencias Agrícolas de la UNJBG en el manejo y uso de la tecnologia informática como internet (instrumento didáctico), y equipos de comunicación audiovisual en los procesos de enseñanza aprendizaje de la educación Universitaria.

La Hipótesis de investigación planteada en el presente estudio fue la siguiente:

El uso de la Internet influye significativamente en el Desempeño académico de los estudiantes de la Facultad de Ciencias Agrícolas de la UNJBG.

\section{MATERIALES Y METODOS}

\section{1. Ámbito de estudio}

El estudio se desarrollo en la Facultad de Ciencias Agricolas de la Universidad Nacional Jorge Basadre Grohmann, teniendo como universo de estudio a las Escuelas Académicas Profesionales de Agronomia, Economia Agraria y Medicina Veterinaria y Zootecnia; asi como la infraestructura informática de la Facultad.

\subsection{Variables de la investigación}

Variable dependiente $(\mathrm{Y})$ : Desempeño académico de los estudiantes de la FCAG
Variables independientes $\left(X_{1}\right)$ :

$X_{1}=$ Uso de internet y equipos informáticos por Estudiantes de la FCAG

$\mathrm{X}_{2}=$ Uso de internet $\mathrm{y}$ equipos informáticos por Docentes de la FCAG

$Y_{1}=\int(X I+X 2)$

\subsection{Población}

La población ( $\mathrm{N}$ ) están constituidos por los Estudiantes de la Facultad de Ciencias Agrícolas que están involucradas las Escuelas Académico Profesionales de Agronomía, Economia Agraria y Medicina Veterinaria y Zootecnia( $\mathrm{N}=557$ alumnos $)$.

\subsection{Muestra}

Método de Muestreo Aleatorio Simple, grado de confianza $95 \%$, valores de p y q 0.5 , error $5 \%$; a partir de los valores señalados se ha determinado $n=384$ muestra, tamaño de muestra ajustada $n "=221$.

\subsection{Diseño estadístico}

El nivel de causalidad entre las variable dependiente e independientes se analizaron utilizando pruebas estadisticas no para métricas chi-cuadrado de Pearson. con un nivel de significancia $\alpha=0,05:$ la interacción de columnas y filas de los cuadros de contingencia, estarán definidos de la siguiente manera:

- Sexo de alumnos con variables de estudio

- Año de estudios con variables de estudio

$$
x^{2}=\sum_{\square=1}^{\infty} \frac{\left.\left(\square_{\square}-\right]_{\square}\right)^{2}}{\square_{\square}}
$$

Donde:

$\mathrm{O}=$ Datos Observados

$\mathrm{Ei}=$ Datos Estimados

$\mathrm{X}^{2}=$ Parámetro Chi-Cuadrado de Pearson

\section{RESULTADOS Y DISCUSIÓN}

\subsection{Uso de internet y equipos informáticos por los} Estudiantes de la FCAG $\left(\mathrm{X}_{1}\right)$

a) Acceso a la computadora por estudiantes de la $F C A G$.

Del análisis practicado sobre la información primaria levantada sobre los alumnos de la Facultad de ciencias Agrícolas se deduce que del $100 \%$ de los encuestados solo el $65 \%$ de ellos manifiestan tener una computadora propia en casa, el $35 \%$ no tiene; y señalan que utilizan los servicios de las cabinas de internet, de sus familiares asi como un bajo porcentaje utilizan las computadoras de la sala de computo de la FCAG. Estas afirmaciones se contraponen con los conceptos planteados por SILVIO (2000), quien señala que, en el contexto de la educación 
superior del siglo XXI, la virtualización es un elemento fundamental en los procesos de enseñanza aprendizaje, lo cual se traduce en el desarrollo de la investigación, extensión y gestión misma en el estudiante.

De los que tienen computadoras propias, el $62 \%$ son del tipo tradicional (PC), el 16\% cuentan con Laptop y el restante no precisa el tipo de computadora debido a que probablemente la que disponen en casa sea una maquina que es compartida entre varios miembros de la misma familia, por lo tanto no necesariamente los consideran comosuyas.

Por otra parte, se desprende que, de los alumnos que respondieron tener una computadora en casa, el $45 \%$ señala estar conectado a algún proveedor de internet, el $55 \%$ restante no tiene conexión, y de los que están conectados, el $47 \%$ de estos indican que tienen una conexión a internet de manera inalámbrica y el $53 \%$ restante, tiene una conexión a internet por cable, es decir, servicio prestado por alguna empresa formal (Telefónica, Claro, otros).

Según el BANCO MUNDIAL (2008), los usuarios de internet (por cada 100 personas) al año 2008, son de la siguiente manera: Argentina 31.3, Chile 32.5, Bolivia 10.8. Brasil 37.5. Perú 24.7, comparados a Japón y Estados Unidos los indicadores son 75.2 y 75.8 respectivamente; es decir a nivel latino América, solo superamos a Bolivia, el resto de paises están con un indicador por arriba del Perú.

\section{b) Utilización de la sala de computo por estudiantes de laFCAG}

De este análisis se desprende que solo el $48 \%$ de los encuestados indican que utilizan la sala de computo de la FCAG, siendo la frecuencia de uso, dos veces por semana, una vez por semana, y hasta una vez al mes, frente al $52 \%$ de los encuestados que señalan que no utilizan la sala de cómputo de la FCAG.

El uso limitado de las computadoras de la FCAG podría deberse a que estos son maquinas desfasadas tecnológicamente, es decir, de menor velocidad de procesamiento o que estas maquinas no cuentes con los servicios de software deseados por los alumnos. Estas afirmaciones se evidencian mas cuando del análisis desarrollado se desprende que el $83 \%$ de los estudiantes de la FCAG afirman que los equipos de cómputos de la FCAG no están debidamente conservados y actualizados, lo cual contraviene las afirmaciones de, MIQUEL(1998),

INTERNET es una poderosa herramienta para ayudar a la difusión del conocimiento y la educación, de hecho es una de las mayores fuentes de información disponibles. Se dice que estamos en la era de la comunicación y el conocimiento, de ahi la importancia de esta red de redes que actualmente se extiende por todas partes del mundo. reduciendo considerablemente el tiempo y esfuerzo empleado en la búsqueda del saber y la información.

Aplicando una prueba estadística de Chi-cuadrado de Pearson para analizar la existencia de una relación de causalidad entre la utilización de algún software y el año de estudios de los alumnos de la FCAG, el análisis indica que existe una relación a un nivel de significancia del $5 \%$, mostrando una relación directa entre el año de estudios de los alumnos de la FCAG con la utilización limitada de software de carácter básico.

\section{c) Uso de buscadores y navegadores por los estudiantes delaFCAG}

No obstante relacionado a la existencia de una gran variedad de navegadores, los estudiantes de la FCAG indican que utilizan en un $51 \%$ el navegador Internet Explore, 26\% Google Chrome y 16\% Mozilla Firetox. desconociendo otros navegadores como Safari, Netscape.

Respecto al tipo de buscadores que utilizan los estudiantes de la FCAG, entre los analizados: Google, Rincón del Vago, Monografias, AltaVista, Yahoo, relacionados al género de los estudiantes; los varones $(51 \%)$ son los que más utilizan los buscadores señalados, con respecto a las mujeres $(49 \%)$, siendo el Google el más preferido seguido de monografías: la frecuencia de uso de otros buscadores es poco significativo. Analizando la relación de causalidad entre ambas variables señaladas a través de la prueba estadistica Chi-cuadrado de Pearson, el resultado señala que existe una relación directa entre el uso de buscadores y el género de los estudiantes, siendo los estudiantes varones los que tienen mayor predisposición para el uso de internet.

\section{d) Sistema de comunicación que utilizan los estudiantes por internet en tiemporeal}

Los estudiantes, indistintamente al año de estudio. tienen como sistema de comunicación en tiempo real con sus pares en orden de importancia el Messenger con $87.3 \%$, Yalıoo $2.3 \%$ y otros $8.1 \%$. Entre el género femenino y masculino de los estudiantes, el género femenino usan el Messenger. En suma el $92 \%$ de estudiantes utiliza alguno de los sistemas de comunicación por internet en tiempo real, mas no ocurre Jo mismo cuando se trata del uso de software básico, lo cual hace suponer que en el nivel de estudios secundarios y universitarios no existe una exigencia a favor al uso de estas herramientas informáticas que están estrechamente relacionados a la calidad de la enseñanza aprendizaje de los estudiantes; no obstante que, FERRES y MARQUES (1996), señalan que, los estudiantes podrian utilizar en mayor o menor medida estas capacidades comunicativas que proporciona Internet, para intercambiar correspondencia electrónica mediante correo electrónico con estudiantes de otros lugares, de esta manera conocen otras realidades y practican otros idiomas; también podrían desarrollar Proyectos cooperativos, es decir, los alumnos de diversos centros realizan proyectos conjuntos coordinando su trabajo a través del correo electrónico; también se pueden desarrollar debates de alumnos. La realización de debates entre alumnos de diversos centros y/o países puede constituir en otra actividad de gran riqueza educativa. 


\subsection{Uso de internet y equipos informáticos por docentes de la FCAG $\left(\mathrm{X}_{2}\right)$}

La influencia del uso de internet en el rendimiento académico de los estudiantes de la FCAG, no solo está relacionada al grado de manejo de estas tecnologías de información y comunicación (TIC) por parte de los estudiantes. sino también en la medida que estas tecnologías sean de uso para enseñanza por parte de los profesores de la Universidad.

Del análisis efectuado sobre la información de campo levantada para la investigación. se tiene que, solo el $23 \%$ de los estudiantes señalan que los profesores de la Facultad utilizan material de enseñanza preparada en computadora y que estos son aplicados hacia ellos; el $72^{\circ} \%$ señala que los profesores utilizan material preparada en computadora en algunas veces, es decir una o dos veces durante el semestre académico; linalmente el $5 \%$ de los estudiantes señala que los profesores nunca utilizan material didáctico preparado en computadora.

Et hecho de que los profesores de manera general, en la Faculad de Cicncias Agricolas no estén utilizando las tecnologias de informacion y comunicacion, esta relacionado directamente a que la Faculad no cuenta con los equipos informáticos y audiovisuales suficientes, por tanto los profesores estan obligados a utilizar métodos tradicionales que no contribuyen a un mejor aprovecham iento académico de los estudiantes.

Aplicando la prueba estadistica Chi-cuadrado de Pearson para determinar la relacion entre el género de alumnos. y si los profesores consultan algunas páginas web para ampliar o reforzar el contenido de las asignaturas, del analisis se desprende que existe una relación de causalidad con un nivel de significancia del $5 \%$.

\subsection{Desempeño acadénico de los Estudiantes de la Facultad de Ciencias Agricolas ( $\left.Y_{i}\right)$}

LIBKIND (2002), señala que la revolución en las comunicaciones que supone Internet impacta en el acceso a la información en todas las áreas del conocimiento, permite anular distancias geográticas. disminuye el costo de la educación, multiplica el acceso a la información. facilita el diálogo entre pares y genera nuevos paradigmas en educación: no obstante el aula lleva 3000 años resistiendo posibles competidoras. Fsta alirmación se sustenta en los resultados del presente estudio. donde el $98 \%$ de los estudiantes tienen una percepción positiva de la influencia de internet en su formación profesional.

Un estudio señalado por M.CHI, el al. (1989), sobre la retención de los contenidos en relación al método de aprendizaje indica que:

$\begin{array}{lc} & \text { Retención } \\ \text { Leer } & 10 \% \\ \text { Ver } & 20 \% \\ \text { Escuchar } & 30 \% \\ \text { Very Escribir } & 50 \% \\ \text { Colaborar } & 70 \% \\ \text { Hacer } & 80 \%\end{array}$

Por tanto, de la presente investigación. se puede deducir que el uso de internet por los estudiantes podria permitir lograr la máxima retención señalada por el Autor, por que las herramientas que ofrece internet permiten interactuar simultaneamente con todos los atributos señalados por el autor.

Un análisis realizado sobre la información historica (05 últimos años) existente en la área de registros académicos de la FCAG, se ha determinado que. el aprovechamiento de los estudiantes durante su formacion profesional, no es constante: por una razón aún no investigada, el rendimiento de los estudiantes sufre una abrupta caida en los dos últinos años (ver Figura 01).

Recientemente la Escuela de Fconomia Agraria a través del proyecto "Fomento a la Investigacion Económica de la UNJBG", financiado con recursos provenientes del canon minero, han logrado una implementacion con los equipos informáticos y audiovisuales sulicientes y de ultima generación. Ios mismos que aim no estan en su plena operacion.

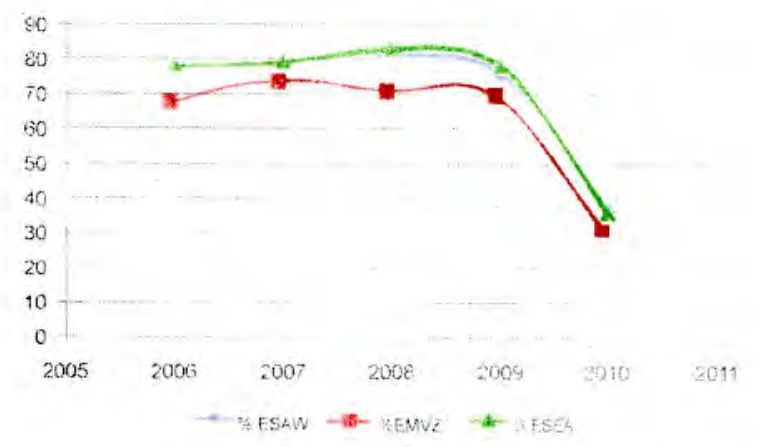

Figura. 01. Porcentaje de alummos aprobados por escuela profesional de la FCAG

Finalmente. se ha realizado un análisis estadistico no para métrica (chi-cuadrado de Pearson) con un nivel de significancia de 0.05 . entre el año de estudios de los alumnos de la FCAG, respecto a $5 \mathrm{u}$ percepción si la utilización del internet y equipos audiovisuales influyen en su formación profesional del analisis se desprende que si existe una relación de causalidad (Tabla $N^{\circ} 01$ ). con lo que queda demostrado la hipotesis de la presente investigación.

Tabla No 01: Pruebas de chi-cuadrado

\begin{tabular}{lccc}
\hline & Valor & gl & $\begin{array}{c}\text { Sig. Asintótica } \\
\text { (bilateral) }\end{array}$ \\
\hline $\begin{array}{l}\text { Chi-cuadrado de } \\
\text { Pearson }\end{array}$ & $74.822(a)$ & 28 & .000 \\
$\begin{array}{l}\text { Corrección por } \\
\text { continuidad }\end{array}$ & & & \\
\end{tabular}

$\begin{aligned} & \text { Razón de } \\ & \text { verosimilitudes }\end{aligned} \quad 66.763 \quad 28$

Asociación lineal por lineal

$\mathrm{N}$ de casos válidos 


\section{CONCLUSIONES}

1. El $65 \%$ de los estudiantes de la Facultad de Ciencias Agricolas, señalan tener computadoras propias y de estos solo el $47 \%$ están conectado al servicio de internet, el restante utiliza internet en cabinas públicas, amigos y en la sala de cómputo de la FCAG; con respecto al uso de equipos informáticos para su formación profesional, el $67 \%$ de los estudiantes indican utilizar solo procesador de textos y hoja de cálculo y solo el $5 \%$ saben utilizar paquetes estadísticos.

2. El $23 \%$ de los estudiantes señalan que los profesores de la FCAG utilizan siempre internet y equipos informáticos para preparar material de enseñanza en clase.

3. Para contrastar la hipótesis de la investigación, se plantearon las hipótesis alternativos de la siguiente manera:

$\mathrm{H} 0$ : El uso de internet no influye significativamente en el desempeño académico de los estudiantes de la Facultad de Ciecnias Agricolas de la UNJBG.

$\mathrm{HI}$ : El uso de internet influye significativamente en el desempeño académico de los estudiantes de la Facultad de Ciecnias Agrícolas de la UNJBG.

Aplicando la prueba estadística de chi-cuadrado de Pearson, con un nivel de significacncia $\infty=0.05$, se tiene que el estadístico calculado es $\mathrm{sig}=0.00$; por lo tanto, el hecho que sig $<0.05$ nos permite rechazar la hipótesis nula y aceptar la hipótesis alternativa; por tanto se concluye que el uso de internet influye significativamente en el desempeño académico de los estudiantes de la Facultad de Ciencias Agrícolas de la UNJBG.

\section{REFERENCIAS BIBLIOGRÁFICAS}

Ferrés, Joan y Marques, Pere (1996). Comunicación Educativa y Nuevas Tecnologías. Pp. 217-229. Barcelona: PRAXIS.

Libkind Alejandro (2002). Papel de internet en la Educación Superior y Continua, 2do Congreso Virtual Iberoamericano de Informática Médica Nov. 4 - Nov. 30,2002 .

Oliver, Miquel (1998). "La videoconferencia en el campo educativo: técnicas y procedimientos". Comunicación y Pedagogia, $\mathrm{n}^{\circ} 151$, pp. 47-51" Barcelona.

Piscoya Hermoza, L.(2006). Metodología de la Investigación Científica, AF A Editores trillas, Lima.

Risp A. Marquez (1981). La Revolución de la información, Aula Abierta Salvat. Colección Salvat TC. Temas Clave. Tomo 99 PP 56,57. Salvat Editores, España.

\section{Correspondencia:}

Francisco Teodoro Condori Tintaya. Ciudad Universitaria Fundo "Los Granados" Av. Miraflores s/n. Tacna. Perú

fracontintaya@yahoo.es 